\title{
Identification of potential HIV restriction factors by combining evolutionary genomic signatures with functional analyses
}

\author{
Paul J McLaren ${ }^{1,2+}$, Ali Gawanbacht ${ }^{3 \dagger}$, Nitisha Pyndiah ${ }^{4 \dagger}$, Christian Krapp ${ }^{3 \dagger}$, Dominik Hotter ${ }^{3}$, Silvia F Kluge ${ }^{3}$, \\ Nicola Götz ${ }^{3}$, Jessica Heilmann ${ }^{3}$, Katharina Mack ${ }^{3}$, Daniel Sauter ${ }^{3}$, Danielle Thompson ${ }^{4}$, Jérémie Perreaud ${ }^{4}$, \\ Antonio Rausell ${ }^{2,4}$, Miguel Munoz ${ }^{4}$, Angela Ciuffi ${ }^{4}$, Frank Kirchhoff ${ }^{3^{*}}$ and Amalio Telenti ${ }^{{ }^{*}}$
}

\begin{abstract}
Background: Known antiretroviral restriction factors are encoded by genes that are under positive selection pressure, induced during HIV-1 infection, up-regulated by interferons, and/or interact with viral proteins. To identify potential novel restriction factors, we performed genome-wide scans for human genes sharing molecular and evolutionary signatures of known restriction factors and tested the anti-HIV-1 activity of the most promising candidates.

Results: Our analyses identified 30 human genes that share characteristics of known restriction factors. Functional analyses of 27 of these candidates showed that over-expression of a strikingly high proportion of them significantly inhibited HIV-1 without causing cytotoxic effects. Five factors (APOL1, APOL6, CD164, TNFRSF10A, TNFRSF10D) suppressed infectious HIV-1 production in transfected 293T cells by $>90 \%$ and six additional candidates (FCGR3A, CD3E, OAS1, GBP5, SPN, IF/16) achieved this when the virus was lacking intact accessory vpr, vpu and nef genes. Unexpectedly, over-expression of two factors (ILIA, SP110) significantly increased infectious HIV-1 production. Mechanistic studies suggest that the newly identified potential restriction factors act at different steps of the viral replication cycle, including proviral transcription and production of viral proteins. Finally, we confirmed that mRNA expression of most of these candidate restriction factors in primary CD4+ T cells is significantly increased by type I interferons.

Conclusions: A limited number of human genes share multiple characteristics of genes encoding for known restriction factors. Most of them display anti-retroviral activity in transient transfection assays and are expressed in primary CD4+ T cells.
\end{abstract}

Keywords: Host restrictions factors, Evolutionary genomics, HIV-1

\section{Background}

Antiretroviral innate defense genes such as TRIM $5 \alpha$, APOBEC3G, BST2/Tetherin, and SAMHD1 exhibit characteristic evolutionary signatures of powerful selective pressures reflecting a long-standing evolutionary arms race between the host and viral pathogens [1]. A second common characteristic of these genes is that they are interferon-inducible and differentially expressed during HIV infection [2,3]. Furthermore, they frequently interact

\footnotetext{
*Correspondence: frank.kirchhoff@uni-ulm.de; atelenti@jcvi.org

${ }^{\dagger}$ Equal contributors

${ }^{3}$ Institute of Molecular Virology, Ulm University Medical Center, 89081 UIm, Germany

5. Craig Venter Institute, La Jolla, CA 92037, USA

Full list of author information is available at the end of the article
}

directly with viral proteins, either to exert their antiviral activity or because they are targeted by viral antagonists [4-6]. Thus, evolutionary and molecular characteristics, such as positive selection in primate genomes, differential expression during infection, and interaction with viral components might constitute a distinct signature of genes endowed with antiviral activity.

We leveraged the availability of complete genome sequences of several primate species (human, chimpanzee, gorilla, orangutan, macaque, marmoset, tarsier, bushbaby, and mouse lemur) to perform a genome-wide screen for genes carrying the signatures of known host restriction factors. To address this, we examined which human genes that are differentially expressed during 
HIV-1 infection, and/or encode host factors interacting with viral proteins have also been subject to diversifying selection during primate evolution. Candidates carrying the most promising combined signatures were examined for their effects on different steps of the HIV-1 replication cycle. We emphasized the confirmation of the IFN-induced nature of the candidates, their significant expression in HIV-1 target cells, the efficient reduction of infectious virus production in the over-expression screen, and a particular emphasis on genes that affected the infectiousness of HIV-1 more severely than viral gene expression and/or showed some specificity for the LTR promoter. The combination of bioinformatics criteria with a broad functional screen allowed bringing a large data set of genes to a manageable list of candidates for further analyses. Our results demonstrate that over-expression of a surprisingly high proportion of these genes inhibits infectious HIV-1 production and suggest that the viral accessory proteins $\mathrm{Vpr}, \mathrm{Vpu}$ and/or Nef may diminish the antiviral effect of some of these cellular factors.

\section{Results}

\section{Genes that are induced during HIV-1 infection have a distinct evolutionary profile}

To examine the contribution of differences in cellular gene expression to viral control, we have previously generated transcriptome data from CD4+ $\mathrm{T}$ cells of untreated HIV-1 infected individuals with different viral loads [3,7]. Additional gene expression data were obtained from published sources on lymph nodes during HIV-1 infection [8]. We also assessed the evidence for evolutionary pressure on all genes by comparing human gene sequences to those of eight additional simian and prosimian species (see methods) and calculated a genewide ratio of non-synonymous $(\mathrm{dN})$ to synonymous $(\mathrm{dS})$ substitutions (gene $\mathrm{dN} / \mathrm{dS}$ ). We found that genes whose expression is positively correlated to viral load in CD4+ T cells $(n=180)$ or induced in lymph nodes $(n=360)$ of HIV-1 infected persons had higher dN/dS values than the genome-wide median for primates $(\mathrm{CD} 4+\mathrm{T}$ cell gene set, $\mathrm{dN} / \mathrm{dS} 0.25$ vs $0.18, p<10^{-5}$, and lymph node gene set $\mathrm{dN} / \mathrm{dS} 0.28$ vs $0.18, p<10^{-5}$ ) (Figure $1 \mathrm{~A}$ ). Genes with $\mathrm{dN} / \mathrm{dS}$ values inflated above the genome-wide reference could either be evolving under more neutral selection, or could have within them certain codons evolving under positive selection that bring up the gene-wide $\mathrm{dN} /$ $\mathrm{dS}$ value. Across these expression datasets, 30 genes upregulated during HIV-1 infection were under positive selection $(\mathrm{dN} / \mathrm{dS}>1)$.

We next examined whether such an evolutionary pattern is also observed in other human infections [9-12]. Compared with the $\mathrm{dN} / \mathrm{dS}$ genome median of 0.18 , genes that are differentially expressed during Dengue virus infection $(\mathrm{n}=158)$, salmonellosis $(\mathrm{n}=205)$, or active tuberculosis $(\mathrm{n}=251)$ generally had higher $\mathrm{dN} / \mathrm{dS}$ values (Dengue, 0.22, $p=0.02$; salmonellosis $0.20, p=$ 0.04; and tuberculosis 0.23, $p=2 \times 10^{-5}$ ) (Figure 1B). A similar pattern was identified for three curated sets of innate immunity genes (http://www.innatedb.ca; $\mathrm{n}=1714$ ) that displayed a statistically significant shift to high $\mathrm{dN} / \mathrm{dS}$ values (all $\mathrm{p}<2 \times 10^{-4}$ ) (Figure 1C). Altogether, these results demonstrate that genes associated with infection and immune response show distinctly higher $\mathrm{dN} / \mathrm{dS}$ values in primates. Although a higher $\mathrm{dN} / \mathrm{dS}$ value could be indicative of relaxed constraint rather than positive selection [13], immune-related genes have consistently been observed to evolve with higher $\mathrm{dN} / \mathrm{dS}$ values and show evidence for codon-specific positive selection [14].

We then extended the analysis to genes described in the NCBI HIV-1 protein interaction database $(n=1251)$ and in the global landscape of HIV-human protein complexes $(n=350)$ [15]. These sets of genes had a significantly lower median $\mathrm{dN} / \mathrm{dS}$ than the genome-wide median for primates $\left(0.14\right.$ and 0.13 vs 0.18 , respectively, both $p<10^{-5}$, Figure 1C). The shift to conservation is comparable to that of other characteristically conserved genes such as those of the OMIM Mendelian disease database $(\mathrm{n}=1289)$ (Figure $1 \mathrm{C})$. This most likely reflects the fact that HIV has to interact with numerous wellconserved cellular factors ("HIV dependency factors") to complete its replication cycle. Although the two HIV interaction datasets were more conserved, 35 genes had $\mathrm{dN} / \mathrm{dS}>1$. As indicated below, some genes under positive selection were common to both the expression and the interaction datasets.

\section{Selection of 30 candidate restriction factors for experimental validation}

Our initial evolutionary scan identified 841 genes (out of a total of 21,389 ) with a dN/dS ratio $>1$ (Figure $2 \mathrm{~A}$ ). We next evaluated in detail the functional profile of this subset by assessing their level of upregulation during HIV-1 infection in vivo, and/or evidence for interaction with viral proteins (see Methods) resulting in an initial shortlist of 57 genes (Additional file 1: Table S1). To confidently assess the genes' evolutionary signatures, we used the codon-level analysis package (codeml) in the Phylogenetic Analysis Using Maximum Likelihood (PAML [16]) software on curated multiple alignments of primate genes (see Methods). This method averages per-codon $\mathrm{dN} / \mathrm{dS}$ estimates across a gene and assesses the evidence for codon-level selection using maximum likelihood estimation. Of the initial 57 candidates, we selected 30 genes for functional analyses based on significant evidence of positive selection at one or more amino acid sites (Additional file 1: Table S1). Eleven of these 30 candidate genes were chosen because they are upregulated during HIV-1 infection, 14 have been shown to interact with viral 

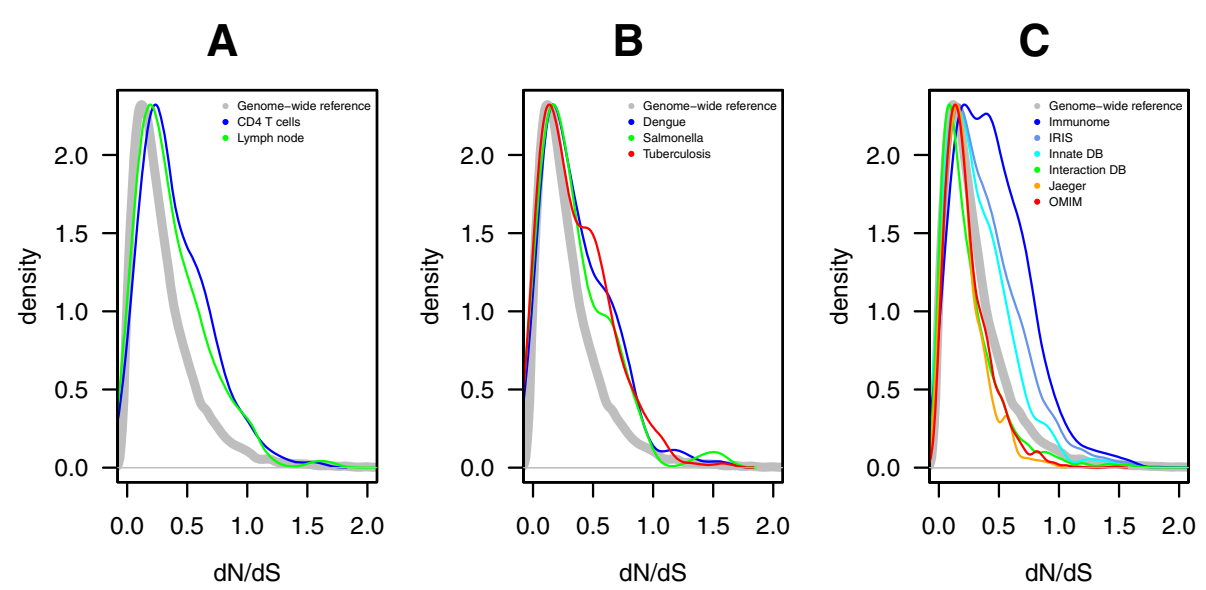

Figure 1 Evolutionary pattern of the protein coding genome in primates. Probability density curves of continuous dN/dS values for genes (A) upregulated in CD4+ T cells and in lymph nodes during infection with HIV-1 in vivo in humans, (B) genes differentially regulated during infection of humans with other pathogens and (C) datasets of human innate immunity genes including: an innate immune specific set (Innate DB), genes curated by the Immunogenetic Related Information Source (IRIS) and a manually curated list of immune genes (Immunome), the NCBI HIV interaction database (Interaction DB), the global landscape of HIV-human protein complexes from Jaeger et al. (Jaeger) [15], and of genes associated with Mendelian disorders in OMIM. The kernel smoothed density estimates (density) of dN/dS values for sets of genes is plotted. The height of the curves is relative to the number of genes with the observed dN/dS values. The genome-wide background dN/dS values for 17,755 genes is shown in grey. Statistically significant differences (Kolmogorov-Smirnov statistics $p \leq 0.05$ ) were observed for distributions towards greater dN/ dS values for genes differentially expressed in CD4+ T cells and lymph nodes during HIV-1 infection, in infection with Dengue virus, Salmonella typhic and tuberculosis, and for distributions towards smaller dN/dS values for genes involved in HIV-1-human interaction datasets and in the catalog of Mendelian disorders.

proteins, and five fulfill both criteria (Figure 2A). As a proof-of-principle, TRIM5 $\alpha$ and APOBEC3G were identified in the screen. Consistent with previous reports $[17,18], B S T 2$ and SAMHD1 reached $\mathrm{dN} / \mathrm{dS}$ ratios of 0.78 and 0.49 , respectively; therefore, they were not shortlisted on the basis of global $\mathrm{dN} / \mathrm{dS}$ ratios, although they displayed codon-specific positive selection. To also consider factors displaying only codon-specific positive selection, we additionally performed a second screen and ranking approach that are described below.

\section{Cell based assays for assessment of restriction}

To examine possible antiviral effects of the candidate genes, we developed cell-based assays to determine their impact on HIV-1 gene expression, virus production and viral infectivity. A total of 27 candidate genes Additional file 1: Table S1 were cloned into a bi-cistronic vector coexpressing the gene of interest and the blue fluorescent protein (BFP) via an internal ribosome entry site (IRES) (Figure 2B). Three of the 30 genes (PARP9, SGOL2, SPAG5) could not be PCR amplified and cloned from cDNA of primary blood cells. 293T cells were cotransfected with the gene expression constructs and HIV-1 NL4-3-based proviral IRES/eGFP constructs. The latter express Nef and the enhanced version of the green fluorescent protein (eGFP) from the same viral RNAs [19]. Thus, the green fluorescence intensity is an indicator of early viral gene expression. To identify possible antagonistic effects of the viral Vpr, Vpu and Nef proteins on the candidate gene products, we screened them for inhibition of a wild-type (wt) NL4-3-based HIV-1 construct and an otherwise isogenic derivate thereof (named $\Delta 3$ ) containing defects in these three accessory genes (Figure 2B). The effect of candidate genes on early LTR-driven gene expression was quantified by fluorescence-activated cell sorting (FACS) at 36 to 48 hours post-transfection (Figure 2C). To monitor the expression of late viral gene products, virus release and infectivity of progeny virions, we assessed the quantity of cell-associated and cell-free p24 capsid antigen and the infectivity of the viral supernatants (Figure 2C). The FACS-based assay allowed us to readily determine the percentage of cells expressing the proviral HIV-1 genome and the cellular candidate genes both alone and in combination (Figure 2D). Importantly, none of the 27 candidate genes caused significant cytotoxic effects or reduced the metabolic activity of the cells under the experimental conditions used to determine their antiviral activity (Additional file 2: Figure S1).

\section{A high proportion of candidate genes display anti-HIV-1 activity}

We found that most candidate genes reduced the frequency of HIV-1-expressing (eGFP+) cells, on average by about 30\% (Figure 3A, Additional file 2: Figure S2 and Additional file 1: Table S1). Expression of four 


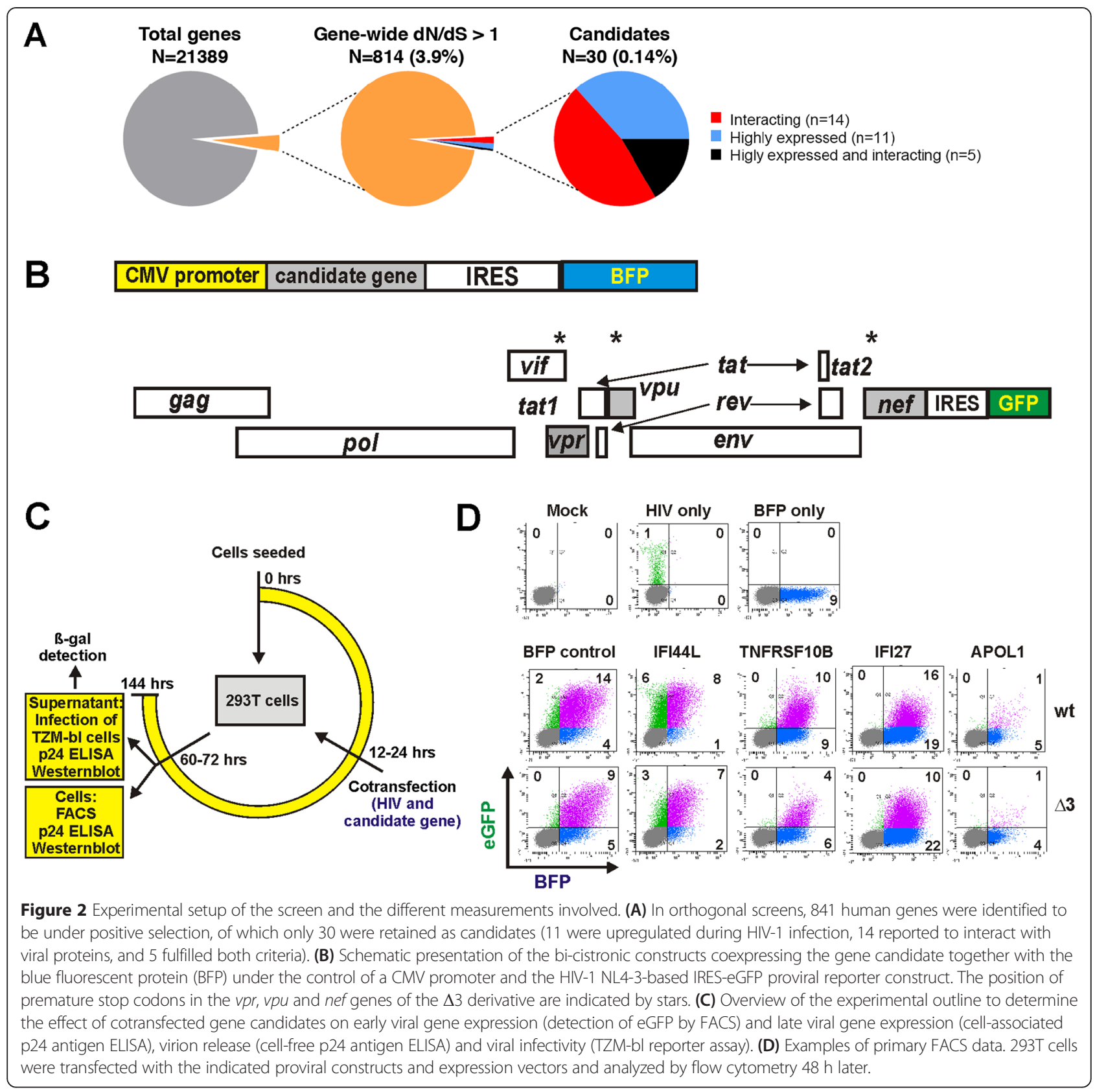

candidate genes (APOL1, APOL3, APOL6 and TNFRSF1OD) achieved more than 50\% inhibition, whereas two genes (IFI44L, SP110) slightly increased the frequency of eGFP+ cells. The effects of the candidate genes on wt and $\Delta 3 \mathrm{HIV}-1$ constructs in the FACS-based assay correlated significantly $\left(\mathrm{R}^{2}=0.93 ; \mathrm{p}\right.$ $<0.0001$ ) (Additional file 2: Figure S3A). This was expected because defects in the $v p r, v p u$ and nef genes should not affect early viral gene expression upon transfection of proviral HIV-1 constructs.

The FACS-based assay also provides an indication for the strength of viral gene expression based on the eGFP mean fluorescence intensity (MFI) since Nef and eGFP are expressed from the same double-spliced bi-cistronic RNA. We found that most candidate genes suppressed LTR-dependent eGFP expression (Figure 3B) and observed a significant but imperfect correlation $\left(R^{2}=0.57\right.$, $\mathrm{p}<0.0001$ ) between the effects of the candidate genes on the percentages of eGFP+ cells and the MFIs of eGFP (Additional file 2: Figure S3B). In addition to the four factors mentioned above (APOL1, APOL3, APOL6 and TNFRSF10D), two additional candidates (OAS1 and IFI16) reduced the MFI of eGFP by more than $50 \%$ (Additional file 2: Figure S2 and Additional file 1: Table S1). 


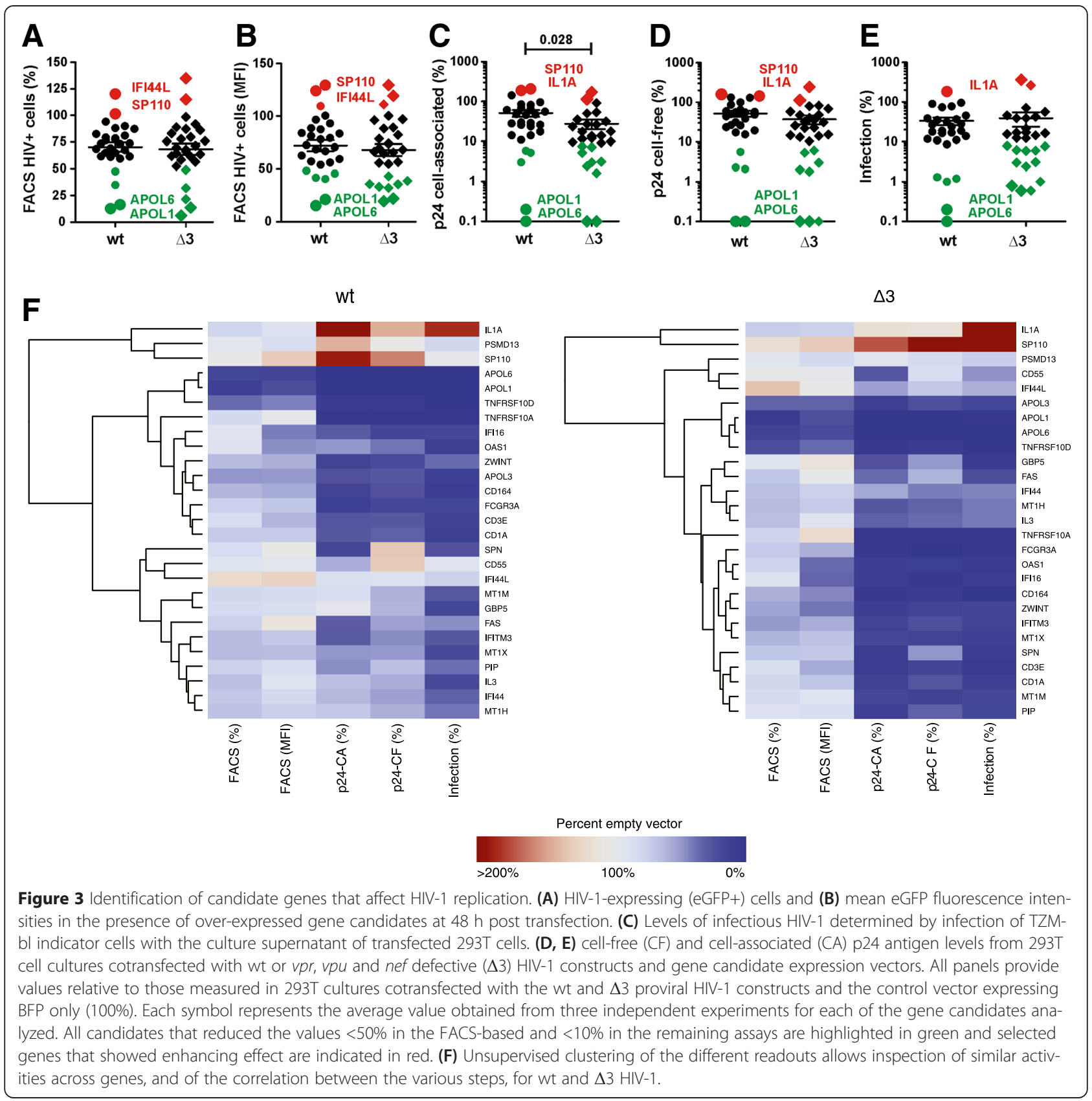

On average, the candidate genes suppressed the levels of intracellular p24 by $49.1 \%$ (Figure 3C) and cell-free p24 production by $48.2 \%$ (Figure 3D) for the wt virus. All candidate genes that reduced p24 antigen expression and production in cells infected with the wt HIV-1 construct also inhibited the $\Delta 3$ virus but the effects were often stronger, i.e. $72.5 \%(\mathrm{p}=0.028)$ reduction of cell-associated and $63.8 \%$ reduction of cell-free p24 production (Figures 3C and 3D). A total of eight cellular factors (APOL1, APOL6, TNFRSF10A, TNFRSF10D, IFI16, CD164, FCGR3A and TRIM $5 \alpha$ ) caused $>90 \%$ reduction of $\mathrm{p} 24$ release by the $\Delta 3$ virus (Additional file 2: Figure S2). We found a significant correlation between the quantities of cell-free and cellassociated p24 antigen $\left(R^{2}=0.62 p<0.0001\right)$ (Additional file 2: Figure S3) and none of the 27 factors analyzed specifically suppressed virion assembly and/or release in the transient transfection assay.

Altogether, over-expression of more than half (15 of 27) of the candidate genes reduced the production of infectious wt HIV-1 by more than $75 \%$ and five factors (APOL1, APOL6, TNFRSF10A, TNFRSF1OD and CD164) achieved $>90 \%$ inhibition (Figure 3E, Additional file 2: Figure S2 and Additional file 1: Table S1). All candidate factors that suppressed the production of infectious wt 
HIV-1 also inhibited the $\Delta 3 \mathrm{HIV}-1$ construct (Additional file 2: Figure S3A). However, the inhibitory effects on the $\Delta 3$ HIV-1 mutant were frequently even more pronounced. In addition to the candidates listed above, six additional cellular genes (OAS1, CD3E, GBP5, FCGR3A, IFI16, and $S P N$ ) achieved $>90 \%$ reduction of infectious HIV-1 production if the virus was lacking intact $v p r, v p u$ and nef genes (Figure 3E, Additional file 2: Figure S2 and Additional file 1: Table S1). Two factors (IL1A and SP110) enhanced the production of infectious HIV-1. The enhancing effect was particularly pronounced for the $\Delta 3 \mathrm{HIV}-1$ construct (Figure 3E, Additional file 2: Figure S2 and Additional file 1: Table S1). Altogether, the levels of viral infectivity and cell-free p24 correlated well $\left(R^{2}=0.66 ; p<0.0001\right)$ although the effects on the former were frequently more pronounced (Additional file 2: Figure S3B). Some factors, such as GBP5 and SPN reduced virion infectivity more severely $(16.4 \%$ and $23.9 \%)$ than the release of p24 antigen $(59.9 \%$ and 132.5\%) (Figure 3F, Additional file 2: Figure S2 and Additional file 1: Table S1) and may thus impair the infectiousness of progeny virions.

To obtain first insights into the possible mechanisms underlying the inhibitory effects, we examined whether the reduced levels of eGFP+ (HIV-1-expressing) cells correlate with the levels of infectious virus production (Figure 3F). We found a significant but imperfect correlation $\left(R^{2}=0.23 ; p=0.0094\right)$ (Additional file 2: Figure $\mathrm{S} 3 \mathrm{~B}$ ) suggesting that reduction of viral gene expression or translation is important but not the only inhibitory mechanisms. For example, the TNFRSF10 genes reduced eGFP expression in the FACS-based assay substantially less effectively than APOL1, APOL3 and APOL6 but achieved a similar reduction in infectious virus production (Figure 3F, Additional file 2: Figure S2 and Additional file 1: Table S1).

\section{Candidate restriction factors inhibit HIV-1 in a dose-dependent manner}

To further verify the antiretroviral activities, we next measured infectious virus yields from $293 \mathrm{~T}$ cells after cotransfection with fixed quantities of wt or $\Delta 3$ HIV-1 NL4-3 proviral vectors and increasing doses of plasmids expressing 13 selected candidate genes. These titration experiments confirmed that APOL1, APOL6, TNFRSF10A, TNFRSF10D, CD164, OAS1, CD3E and FCGR3A inhibit HIV-1 in a dose-dependent manner (Figure 4). The titration analyses also showed that some genes (APOL1, $A P O L 6, C D 164$ and $C D 3 E$ ) reduced the yield of infectious wt HIV-1 slightly less efficiently than of the $\Delta 3$ virus. Interestingly, FAS generally increased infectious HIV-1 production at low expression levels but significantly reduced it at the highest dose of expression plasmids (Figure 4). We also verified that SP110 and IL1A increased the infectious virus yield. Strikingly, IL1A increased infectious virus yield by up to 3.5 -fold for the wt and even up to 7 -fold for the $\Delta 3$ construct. Finally, we examined the effects of the interferon-induced IFI44 and IFI44L. Notably, the latter reduced infectious HIV1 yield more efficiently (Figure 4) although both genes share a significant degree of sequence homology. Altogether, the titration experiments confirmed that many of the newly identified candidate restriction factors suppress infectious HIV-1 production in transiently transfected $293 \mathrm{~T}$ cells.

\section{A high proportion of candidates affect the activity of viral promoters}

The FACS-based assays suggested that some of the candidate factors may reduce the transcription of viral genes. To examine the effect of the candidate factors on HIV-1 promoter activity, we cotransfected 293T cells with a firefly luciferase reporter vector controlled by the HIV-1 LTR, plasmids coexpressing the candidate genes and BFP, and a construct expressing the NL4-3 Tat protein. For comparison, we also analyzed the effects on the herpes simplex virus thymidine kinase (HSV-TK) and cytomegalovirus immediate early (CMV-IE) promoters. Many candidate factors, particularly $A P O L$ family members and FCGR3A encoding the low affinity immunoglobulin gamma Fc region receptor III-A, efficiently suppressed HIV-1 LTR activity (Figure 5). However, the effects were not specific for HIV-1 since these candidate factors also inhibited the activity of the CMV-IE and HSV-TK promoters. Perhaps more notably, several candidate genes, e.g. CD1A, CD3E, GBP5 and SPN had little if any effect on viral LTR activity (Figure 5 ) but still efficiently prevented the production of infectious HIV-1 (Additional file 2: Figure S2). Thus, the results confirmed that reduced viral gene expression is an important but not the only inhibitory mechanism.

\section{A high proportion of candidate genes are interferon- inducible in CD4+ T cells}

The genome-wide screen used gene expression data from in vivo studies in HIV-positive individuals where gene expression was correlated to patient viral load $[3,7]$. To better dissect the general agreement between in vivo data with actual interferon inducibility, we assessed the response of primary CD4+ $\mathrm{T}$ cells from healthy donors to stimulation with interferon alpha, interferon gamma and to cell activation via the $\mathrm{T}$ cell receptor (TCR). The analysis identified a general agreement between in vivo data and interferon alpha gene induction, with the majority of the genes being strongly up-regulated by stimulation (in healthy donors) and by viral replication in $\mathrm{HIV}+$ patients (Figure 6). These data confirm that the in vivo response to HIV infection is 

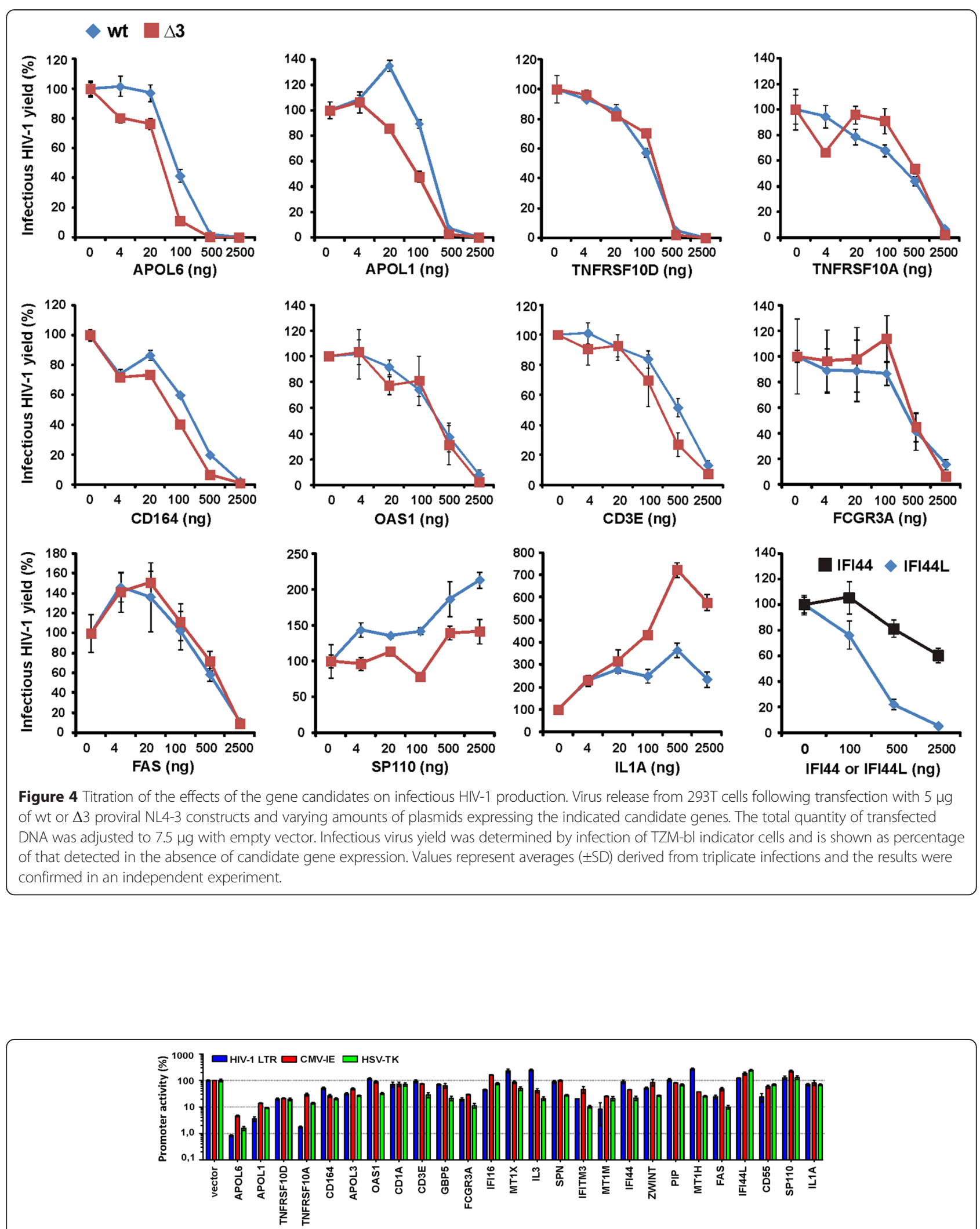

Figure 5 Effect of candidate gene expression on viral promoter activity. Effect of the indicated candidates on the activity of the HIV-1 LTR in the presence of Tat and the CMV-IE and HSV-TK promoters. All data were normalized to the BFP only control construct (100\%) and represent average values $( \pm$ SEM) derived from three transfections. 
greatly driven by the interferon response, although other drivers of immune activation, as exemplified here by TCR stimulation, share parts of the signature. Thus, many of the candidate genes identified in the present study are true interferon-stimulated genes (ISGs) as shown by in vivo and in vitro data and expressed in the main target cells of HIV-1 in infected humans.

\section{Relaxed screening approach ranks genes with potential antiviral activity}

The first screening imposed a hard threshold for inclusion $(\mathrm{dN} / \mathrm{dS}>1)$ to identify genes under (global) positive selection pressure. Use of this global gene metric is a useful link to previous literature; however, tools such as
PAML that assess local signatures of selective pressure and have built-in statistics are becoming a preferred approach. In particular, genes of some known restriction factors (e.g. BST2 and SAMHD1) just show signatures of codon-specific (local) instead of global positive section pressure. Thus, we explored a second genome-wide approach to rank genes using several metrics based on characteristics of innate immunity genes [20]. We initially considered eight parameters associated with innate immunity genes: $\mathrm{dN} / \mathrm{dS}$ value, codon-specific positive selection (PAML), measurements of cross species adaptive evolution, burden of synonymous, missense and non-sense variation, intolerance to functional variation and number of paralogs, as well as the in vivo gene
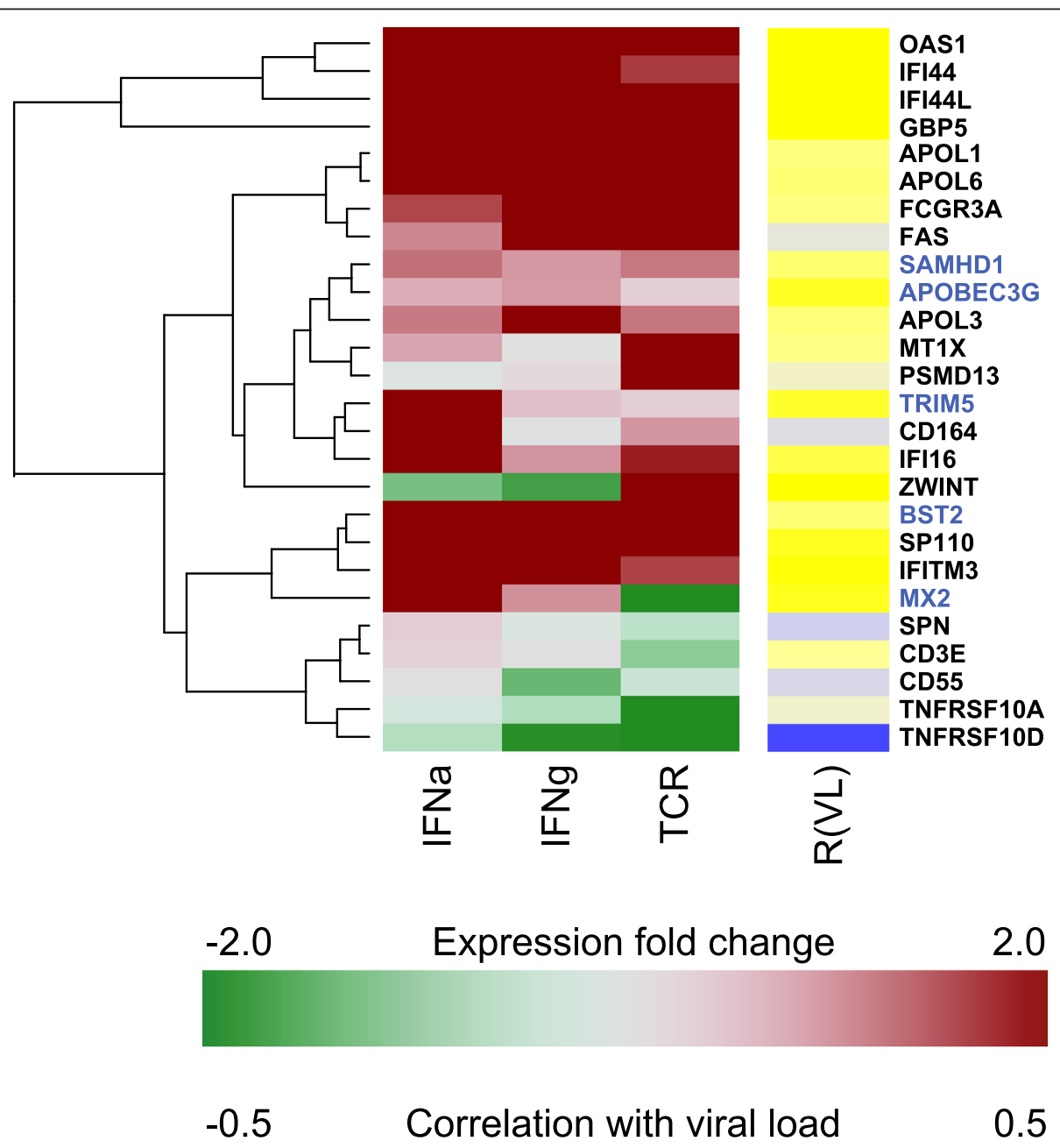

Figure 6 Heat map of expression after stimulation of known HIV-1 restriction factors and gene candidates through the screening approach. On the left, CD4+ T cells from healthy donors were left unstimulated or stimulated with IFNa, IFNy or aCD3/CD28. Expression levels of known restriction factors (blue), and factors identified in the screen (black) were clustered using complete hierarchical clustering. For comparison, the right panel depicts the correlation between gene expression in CD4+ T cells of HIV infected donors with setpoint viral load (R(VL)). Correlation values were taken from [3]. Positive R(VL) values (yellow) indicate an increased gene expression correlates with an increase in patient viral load (log ${ }_{10}$ RNA copies/ml). 
expression response to HIV-1 infection. We trained a model using the set of known HIV restriction factors (APOBEC3G, BST2/Tetherin, MX2, SAMHD1 and TRIM5 $\alpha$ ). Gene parameters were iteratively included/excluded, and the final set of parameters was selected such that the sum of the ranks across the known genes was minimized (see Methods). We present in Additional file 3: Table S2 the top 200 ranked genes. This is a resource that avoids the strict cutoff criteria of $\mathrm{dN} / \mathrm{dS}>1$ to accommodate a more extensive set of gene candidates. Known restriction factors APOBEC3G, TRIM5 $\alpha, M X 2$, SAMHD1 and $B S T 2$ were ranked 2, 5, 125, 277 and 379 among 15,052 protein-coding genes respectively in the final model. Overall, 20 of the 30 candidates identified in the initial screen also ranked above the 95 percentile for the genome in the second approach, and all but one (TNFRSF10D) ranked above the 75 percentile.

\section{Discussion}

In the present study, we utilized features of known antiviral host restriction factors [21], such as evolutionary signatures of positive selection, expression during HIV infection, and interaction with viral proteins, to identify novel cellular factors that inhibit HIV-1. Initially, we performed a canonical genome-wide screen revealing that only a small proportion of the genome (841 of 21389 genes; $3.9 \%$ ) shows evidence for positive selection in primates $(\mathrm{dN} / \mathrm{dS}>1)$. Next, we examined which of these genes are significantly upregulated during HIV-1 infection in vivo and/or encode for cellular factors known to interact with HIV-1 proteins. Based on these selection criteria 30 candidate genes were selected for closer examination and a strikingly high proportion of them suppressed infectious HIV-1 production in transfected 293T cells. To consider the fact that known restriction factors, such as APOBEC3G, BST2 and SAMHD1, show site-specific rather than global evidence for positive selection, we conducted a second screen with more flexible modeling on features of known HIV restriction factors to rank genes without imposing strict thresholds for calling a candidate. All five known restriction factors (APOBEC3G, TRIM5 $\alpha$, MX2, SAMHD1 and BST2) ranked within the top $2.5 \%$ of all protein-coding genes examined (average 1.0\%). The analysis extends previous work from our group [17] and work of Meyerson et al. [22] that searched for signatures of positive selection among previously described HIV host factor genes. The current genome-wide analyses allowed identification of additional genes sharing characteristics of known restriction factors, a useful basis for the identification of novel intrinsic antiretroviral cellular factors.

To evaluate possible antiviral effects of the selected genes, we determined their impact on HIV-1 gene expression, virus production and viral infectivity. A set of
27 genes encoding cellular proteins not previously implicated as HIV-1 restriction factors were amplified from primary human blood cells and cloned into a bicistronic vector coexpressing the gene candidate BFP. Over-expression of a striking proportion of candidate genes $(16$ of $27 ; 59 \%)$ reduced the production of infectious wt HIV-1 particles by more than $75 \%$ without causing cytotoxic effects and this number was even higher in the absence of intact $v p r, v p u$ and nef genes (20 of 27; 74\%). For example, genes of the apolipoprotein L gene family (APOL1, APOL3, APOL6) were generally very potent inhibitors. All members of this gene family have rapidly evolved in primates [23], and APOL1 has been shown to confer immunity to Trypanosoma brucei that can be countered by a trypanosome-encoded antagonist [24]. Members of the TNF-receptor superfamily (TNFRSF10A and 10D) were also powerful inhibitors. Notably, TNFRSF10A was also identified as one of the most potent anti-HIV-1 factors among 380 type I interferon-stimulated genes in a previous study [25]. Another potent inhibitor of HIV-1 was CD164 (also known as endolyn) a cell adhesion molecule that interacts with CXCR4 [26]. Furthermore, the high activity of OAS1 is noteworthy because this interferon-induced factor is known to generate 2',5'-oligoadenylates which activate RNase L thus causing viral RNA degradation and inhibition of viral replication [27]. Notably, OAS1 affected the percentage of HIV-1 infected (eGFP+) cells less severely than the mean fluorescence intensity of eGFP expression, which would be expected in the case of RNase L mediated viral RNA degradation. A similar phenotype was observed for IFI16, which is involved in transcriptional regulation and plays a role in the sensing of intracellular viral DNA [28,29]. More recently, IFI16 has been reported as a key player in inducing pyroptosis in abortively infected quiescent CD4+ T cells, thereby contributing to the massive depletion of $\mathrm{CD} 4+\mathrm{T}$ cells observed during HIV infection [30,31]. Accumulating evidence suggest that restriction factors, such as TRIM $5 \alpha$ [32] and BST2/tetherin [33-35] may also act as immune sensors and direct anti-HIV effects of IFI16 warrant further study. Conversely, some genes that displayed antiHIV activity are known to be involved in signaling, both in various cancers (CD164, TNFRSF10A/D, ZWINT, MT1X, IL3) and immune or $\mathrm{T}$ cell activation (GBP5, CDIA). Thus, these genes may not inhibit HIV-1 directly but could also induce cellular responses associated with the expression of other innate immunity factors. This possibility is in agreement with our observation that some candidate factors inhibited various viral promoters. It is also noteworthy, however, that $293 \mathrm{~T}$ cells lack many intact immune signaling pathways and did not express detectable levels of interferon after transfection with constructs expressing the various candidate factors. Further studies 
will be necessary to clearly distinguish between direct and indirect anti-HIV effects.

Two of the 27 cellular genes examined (SP110 and IL1A) enhanced production of infectious HIV-1. SP110 is part of a leukocyte-specific nuclear body component that may also play a role in the regulation of gene transcription. Mutations in SP110 are associated with immunodeficiency [36]. It has been reported that this factor stabilizes EBV mRNAs and enhances lytic viral gene expression [37]. The strongest enhancing effects on infectious virus production (up to 7 -fold in dosedependency studies) were observed for IL1A. This cytokine plays a key role in inflammation and the regulation of the immune response. It has been reported that HIV1-exposed seronegative sex workers show reduced levels of IL1A expression in their genital mucosa [38]. Thus, the present results confirm and expand previous data supporting that these cellular factors may enhance viral infection and clearly warrant further investigation.

In most cases, the effect of the candidate restriction genes in the FACS-based assays, intra- and extracellular p24 levels and infectious virus production correlated with one another, suggesting that reduced viral gene expression and/or translation are responsible for the inhibitory activity of the cellular factors rather than effects on virus release or virion infectivity. In support of this, preliminary data using LTR-luciferase reporter constructs suggest that many candidates suppress LTR activity (Figure 5). Nonetheless, our results also provide evidence that some candidate genes target different steps of the viral life cycle. For example, the guanylate-binding protein 5 (GBP5) and the mucin-like factor SPN (also known as leukosialin, sialophorin or CD43) reduced viral infectivity more severely than the levels of cell-free p24 antigen and may thus specifically reduce virion infectivity. GBP5 is a member of the dynamin superfamily of GTPases, which have been implicated in protection against various pathogens $[39,40]$. Another factor (IFITM3), that was identified by our screen and reduced infectious virus production, was recently shown to be incorporated into HIV-1 virions and to impair their infectiousness [41,42]. SPN regulates CD4+ T cell proliferation and migration and shows reduced expression and aberrant glycosylation in HIV-1-infected individuals $[43,44]$. Thus, it is conceivable that GBP5, SPN and IFITM 3 may affect virion infectivity although the underlying molecular mechanisms of the former two remain to be determined.

Some known host restriction factors, i.e. APOBEC3G, Tetherin and SAMHD1, were initially discovered because they are counteracted by viral accessory proteins $[4,5]$. We found that the absence of intact $v p r, v p u$ and $n e f$ genes increased the inhibitory effects of several candidate factors (e.g. APOL1, APOL6, CD164, GBP5, SPN,
CD3E, ZWINT and PIP) on p24 and infectious virus production. However, the differences were generally modest because even wt HIV-1 was efficiently inhibited at high expression levels of the candidates. Notably, overexpression of known restriction factors, such as tetherin, can also overcome the viral antagonists. Thus, it remains to be clarified whether the lower intrinsic infectiousness of the $\Delta 3 \mathrm{HIV}-1$ construct compared to the wt virus or specific antagonistic effects of the accessory $\mathrm{Vpr}, \mathrm{Vpu}$ and/or Nef are responsible for the observed differences in susceptibility to inhibition by some candidate factors.

Not all recognized restriction factors would have been identified under the criteria of the evolutionary genetic screen because of the criteria that imposed a dN/dS $>1$. We could however demonstrate that alternative approaches that model several characteristics of innate immunity genes ranked all known restriction factors and most of the novel candidates among the top 5\% genes in the genome. Overall, genes that emerged from the screen were strongly induced by interferon alpha. These results indicate that they are bona fide interferon-stimulated genes (ISGs) that are possibly endowed with antiviral activity. Our ongoing studies aim to further define the antiviral mechanisms of the most potent and specific candidate genes as well as their possible interaction with the accessory viral proteins.

In summary, we show that the number of cellular genes showing evolutionary and functional characteristics similar to known host restriction factors is limited. Interferon treatment induced expression of most of these genes in CD4+ T cells and over-expression of a strikingly high proportion of them in transfected 293T cells inhibited the production of infectious HIV-1. These results are consistent with the observation that interferon administration, likely through the action of yet to be characterized interferon stimulated genes, inhibits HIV and related primate lentiviruses $[45,46]$. The underlying mechanisms and the relevance of these potential restriction factors to the control of HIV-1 replication in relevant viral target cells and in vivo remain elusive and further characterization of these genes may improve our understanding of cellular defense mechanisms.

\section{Experimental Procedures}

\section{Expression and protein interaction datasets}

We used transcriptome data from purified CD4+ T cells from 128 HIV-1 infected seroconverting individuals representing the complete range of viral setpoint [3] using Illumina Human HT-12 V3 BeadChip arrays (Illumina). Patient spVL was calculated as the average of all $\log 10$ transformed viral load measurements (RNA copies/ml) after the acute phase of infection and prior to disease progression $(\mathrm{CD} 4<350$ cells $/ \mathrm{ml})$ or the initiation of ART as described [47]. 
Association analysis was performed regressing expression level of each gene on patient spVL controlling for gender, age, CD4+ $\mathrm{T}$ cell viability and batch effect. In addition, we compiled data from transcriptome analysis of other active infections that used comparable genomewide technologies (Illumina, Affymetrix and other arrays containing $>20,000$ human transcripts): the transcriptome of lymph nodes of HIV-infected individuals [8], and expression analyses of peripheral blood from individuals with tuberculosis [9], typhoid fever [10], and dengue [11,12]. Based on the HIV host transcriptome studies, we defined post-hoc a HIV-1 expressed gene set that included 527 genes upregulated during in vivo infection. The NCBI HIV-1-human protein interaction database (www.ncbi.nlm.nih.gov/RefSeq/ HIVInteractions/) and the "global landscape of HIV-human protein complexes" [15] were used to evaluate interactions between HIV-1 and cellular factors. InnateDB (www.innatedb.ca), a publicly available database of the genes, proteins, experimentallyverified interactions and signaling pathways involved in the innate immune response was included to evaluate the general profile of genes participating in the response to microbial infection. Genes were extracted from the OMIM Gene Map (www.ncbi.nlm.nih.gov/sites/entrez? $\mathrm{db}=\mathrm{omim}$ ), and a dedicated set was created by including genes associated with a disease and $\mathrm{C}=$ confirmed observed in at least two laboratories or in several families.

\section{Codon alignments of primate orthologous genes}

Genome-wide codon alignments of orthologous genes for up to nine simian and prosimian species (human, chimpanzee, gorilla, orangutan, macaque, marmoset, tarsier, bushbaby, and mouse lemur) were collected from Ensembl v57. First, one-to-one orthologous primate gene trees were gathered from the Ensembl Compara database by extracting the sub-tree corresponding to all 'one2one' primate orthologs of each human gene, as annotated by Compara's gene tree reconciliation and homology annotation method [48]. These one-to-one primate gene trees, which included branch lengths in units of expected nucleotide substitutions per site, were used to guide the rest of the analysis.

It has been shown that errors in sequencing, annotation and alignment can lead to excessive false positives in downstream evolutionary analyses [49]. As a result, efforts were made to avoid including possibly erroneously sequenced or aligned bases in our dataset. Comparison between Compara's protein-based alignments and its DNA-based alignments showed that the protein-based alignments often contained missing or alternativelyspliced exons in otherwise highly similar primate orthologs, leading to stretches of erroneously-aligned bases and inflated estimates of evolutionary rates and $\mathrm{dN} / \mathrm{dS}$ (data not shown). In order to avoid such errors, we gathered human-flattened genomic alignments by using exon coordinates from the human Consensus Coding Sequence (CCDS) transcript, or if no CCDS transcript was available, the longest human protein-coding transcript, to extract all available aligned primate DNA sequences from the ' 12 eutherian mammals' DNAbased multiple genomic alignments from Ensembl's Enredo-Pecan-Ortheus pipeline [50]. Low-quality genomic sequence was excluded by masking any nucleotides with a PHRED (or PHRED-equivalent) quality score below 30 (corresponding to an error rate of 1 in 1,000) from the chimpanzee, orangutan, macaque, marmoset, tarsier, bushbaby and mouse lemur source genomes with ' $N$ ' characters; genome quality scores were downloaded from the originating institutions for each genome assembly used in Ensembl v57. Finally, sequences in the generated alignments were cleaned in preparation for codon model analysis by masking out any frameshifting indels (which within these closelyrelated primates are more likely to be the result of sequencing error than true biological indels) and premature stop codons with ' $\mathrm{N}$ ' characters. The precision of the genome-wide values of $\mathrm{dN} / \mathrm{dS}$ was assessed by contrasting the estimates with those from 126 curated alignments [17].

The codeml program from PAML v4.4 was used to evaluate each alignment for evidence of positive selection acting on one or more amino acid sites throughout the evolutionary tree of primates. Specifically, we first used codeml model M0 to infer branch lengths for the evolutionary tree corresponding to each alignment, and then we used those branch lengths as input to models M7 and M8 in order to perform a likelihood ratio test (LRT) for evidence of localized positive selection [51]. P-values were calculated by comparing the LRT statistic to the chisquared distribution with 2 degrees of freedom, and the set of raw p-values was corrected for multiple testing using the Benjamini-Hochberg method [52]. The adjusted p-value for a given alignment represents the overall expected false discovery rate when using that alignment's LRT statistic as a cutoff threshold. Raw and adjusted p-values for the candidate genes are included in Table S1.

\section{Statistics}

The analysis of the differences in distributions used permutation test $(n=100,000)$ with Kolmogorov-Smirnov statistics. Probability density functions for the various gene sets (Figure 1) were plotted assuming continuous $\mathrm{dN} / \mathrm{dS}$ values using kernel density estimates. Statistical analyses and graphical representations were performed by using the $\mathrm{R}$ package (www.r-project.org). Correlation analysis was performed using Pearson $r$ evaluation. 


\section{Expression vectors}

Bi-cistronic pCG expression vectors containing the immediate early promoter of human cytomegalovirus (CMV) coexpressing a candidate gene and BFP via an internal ribosome entry site (IRES) were generated as described elsewhere $[53,54]$. Briefly, the candidates were amplified from human cDNA obtained from primary blood cells by standard PCR reaction using primers introducing flanking $\mathrm{XbaI}$ and $\mathrm{MluI}$ restriction sites for cloning into the expression vector. All PCR-derived inserts were sequenced to confirm their accuracy. Silent mutations were accepted.

\section{Proviral HIV-1 constructs}

Generation of wt HIV-1 (NL4-3-based) proviral reporter constructs containing a functional nef gene followed by an IRES and the eGFP gene and the triple mutant $(\triangle v p u \Delta$ $n e f \Delta v p r)$ have been described previously $[19,54,55]$.

\section{Cells and transfection}

HEK293T cells were maintained in Dulbecco modified Eagle medium (DMEM) supplemented with 10\% FCS (1\% Glutamine, 1\% Penicillin/Streptomycin). A total of $500,000293 \mathrm{~T}$ cells per well were seeded in 6-well plates and transfected with $5 \mu \mathrm{g}$ DNA (1:1 ratio provirus:candidate gene) at a confluence of about $70 \%$ by the calcium phosphate method. TZM-bl cells were kindly provided by Drs. Kappes and Wu and Tranzyme Inc. through the NIH AIDS Reagent Program and were kept in DMEM supplemented with 10\% FCS (1\% Glutamine and 1\% Penicillin/Streptomycin). TZM-bl cells express large amounts of CD4, CCR5 and CXCR4 and contain the $\beta$ galactosidase gene under the control of the HIV-1 promoter.

\section{Infectivity assay}

The yield of infectious HIV-1 was determined by a 96well infection assay using TZM-bl indicator cells. Briefly, 6,000 cells were seeded out in 96-well dishes in a volume of $100 \mu \mathrm{l}$ and infected in triplicate after overnight incubation with 10 or $100 \mu \mathrm{l}$ of virus stocks. Three days later infection was detected using a galactosidase screen kit from Applied Bioscience as recommended by the manufacturer. $\beta$-Galactosidase activities were quantified as relative light units per second (RLU/s) using an Orion II Microplate Luminometer (Berthold).

\section{p24 antigen ELISA}

Nunc immuno maxi sorb surface 96-well plates were coated with a mouse anti-p24 monoclonal (MAK183) antibody (EXBIO) overnight. After blocking, Triton X100 lysed supernatants or cells were transferred to the 96-well plates. The next day, plates were washed and incubated with a polyclonal rabbit anti-HIV-1 p24 antibody (Eurogentec) for $1 \mathrm{~h}$. Next, plates were washed and incubated with a goat anti-rabbit antibody conjugated with horseradish peroxidase (Dianova, 111035-008) followed by the addition of TMB peroxidase substrate. The reaction was stopped with $0.5 \mathrm{M} \mathrm{H}_{2} \mathrm{SO}_{4}$ and OD was measured at $450 / 650 \mathrm{~nm}$.

\section{Flow cytometric analysis}

Flow cytometry analysis of BFP and eGFP reporter expression in $293 \mathrm{~T}$ cells transfected with bi-cistronic vectors coexpressing the gene candidate and BFP together with proviral HIV-1 IRES eGFP was performed on a FACS CANTO II (BD) flow cytometer essentially as described previously [54]. For analysis, the percentage of $\mathrm{BFP} / \mathrm{eGFP}$ double positive cells was determined in relation to BFP positive cells in total. All values were normalized to the sample transfected with a control plasmid coding for no functional protein followed by an IRES BFP cassette.

\section{Cytotoxicity assay}

The potential cytotoxicity of candidate genes was evaluated by MTT assay. 293T cells $(n=20,000)$ were transfected in 96-well format with vectors expressing the candidate genes. After 2 days $10 \mu \mathrm{l}$ of a $5 \mathrm{mg} / \mathrm{ml}$ MTT (3-(4,5-dimethylthiazole-2-yl)-2,5-diphenyl tetrazolium bromide, Sigma \#M2003) solution were added and incubated for three hours. Thereafter cell free supernatant was discarded, formazan crystals were solubilized in $100 \mu \mathrm{l}$ DMSO-Ethanol (1:1) and OD was detected at $490 / 650 \mathrm{~nm}$ to evaluate cell viability.

\section{Promoter reporter assays}

To determine the modulation of viral promoter activity, 293T cells were cotransfected with equal amounts $(2.5 \mu \mathrm{g})$ of firefly luciferase reporter vectors for the HIV-1 long terminal repeat (LTR), HSV thymidine kinase (TK) and CMV immediate early (IE) promoters as well as vectors coexpressing the candidate genes and BFP. The HIV1 LTR promoter was activated by cotransfection of a plasmid $(0.5 \mu \mathrm{g})$ expressing HIV-1 NL4-3 Tat if indicated. $40 \mathrm{~h}$ post transfection, cells were lysed in Luciferase Cell Culture Lysis Reagent (Promega, E1531) and firefly luciferase activity was determined using the Promega Luciferase Assay System (E1501) according to the manufacturer's protocol. The pCMV-IE and pHSV-TK red firefly luciferase reporter vectors were purchased from Thermo Scientific (16156 and 16157, respectively). The 5'-LTR of HIV-1 NL4-3 was inserted into the pGL3 enhancer vector from Promega (E1771) via XhoI/MluI. HIV-1 NL4-3 Tat was cloned into a pCG expression vector via XbaI/MluI. 


\section{RNAseq Analysis}

Resting CD4+ T cells were purified from two healthy blood donors by Ficoll gradient separation followed by negative selection and magnetic separation using the human CD4+ T Cell enrichment kit supplemented with anti-HLA-DR, anti-CD25 and anti-CD69 (Stem Cell Technologies). Cells $\left(10^{6}\right)$ were incubated for 24 hours, either with medium only, or with 100 or $1000 \mathrm{IU} / \mathrm{ml}$ interferon $\alpha$ (Roferon-A, Roche) or interferon $\gamma$ (R\&D Systems), or with $2 \mu \mathrm{g}$ anti-CD3/1 $\mu \mathrm{g}$ anti-CD28 and $100 \mathrm{U} / \mathrm{ml}$ recombinant interleukin-2 (R\&D Systems) to mimic TCR stimulation. After 24 hour incubation, total RNA extraction was performed using Illustra RNAspin mini isolation kit (GE Healthcare) and further processed for mRNA-Seq library preparation (TruSeq RNA sample prep kit, Illumina). $100 \mathrm{bp}$ paired-end sequencing was performed using Illumina HiSeq2000. About 140 mio read pairs were obtained for the samples. Sequencing reads were quality filtered before alignment using Cutadapt [56] and PrInseq [57]. Cleaned sequencing reads were aligned to a build genome using SOAPsplice. Gene expression was assessed by determining the number of mapped reads per gene using HTSeq-count tool, followed by DESeq $\mathrm{R}$ package normalization. An additional normalization step taking into account the gene coding length was performed so that the resulting counts could be compared across samples for a given gene, and also between genes.

\section{Ranking screen}

A set of 15,052 protein-coding genes was annotated with nine parameters informative for response to HIV-1 infection or evolutionary history. Parameters included in vivo response to HIV-1 infection (i.e. expression correlation with viral load [3], primate $\mathrm{dN} / \mathrm{dS}$ value, codon-specific positive selection (PAML), measurements of cross species adaptive evolution (the McDonald-Kreitman value comparing human sequence to chimp, orangutan or rhesus [58], burden of synonymous, missense and non-sense variation, intolerance to functional variation (rare variant intolerance score obtained from [59]) and number of paralogs. Burden of synonymous, missense and non-sense variation was taken from exome sequence data on $\sim 6,300$ individuals sequenced as part of the NHLBI exome sequencing project (http://evs.gs.washington.edu/EVS/). Burden was calculated per gene as the total number of variants in each class divided by gene length. Genes were ranked for each parameter (with top ranked genes showing high positive correlation with HIV viral load and high levels of within and between species diversity) and the ranks were summed giving equal weight to each measurement. Beginning with all measurements, parameters were iteratively dropped and re-included (individually and in combination) in the ranking and the final model was selected based on the set of parameters that optimized the ranking of known HIV-1 restriction factors. The final set of parameters included in vivo response to HIV-1 infection, $\mathrm{dN} / \mathrm{dS}$ value and evidence for codon-specific positive selection (PAML P-value).

\section{Additional files}

Additional file 1: Table S1. Genes selected on the basis of evolutionary history in primates, expression during HIV-1 infection in vivo, or issued from the HIV-1 interaction database. Because of errors in sequencing, annotation and alignment can lead to excessive false positives in downstream evolutionary analyses. Therefore, we assessed dN/dS estimates using both Ensembl Compara's protein-based alignments (columns c_m0 and c_m), and DNA-based alignments of primate sequences generated from genomic DNA alignments (column g_m0). The " $\mathrm{m0}$ " columns (c_m0 and g_m0) show gene-wide $d N / d S$ values estimated using the SLR software under a one-ratio model (i.e. one $\mathrm{dN} / \mathrm{dS}$ value per gene), while the " $\mathrm{m}$ " column (c_m) shows the mean across the gene of all codon-specific dN/dS estimates using SLR's sitewise model

(Massingham and Goldman, 2005). The raw and adjusted p-values for sitewise positive selection, measured using the so-called 'site' test for positive selection (Yang, 2007), are included (column paml_pval, and Benjamini-Hochberg FDR-adjusted: paml_adj_pval). In column

"Association", E=differentially expressed during HIV-1 infection in vivo, I= included in the NCBI HIV-1. interaction database. The effect of the candidate genes on NL4-3 and D3 HIV-1 is indicated in the following columns. Each value represents the average values $(n \geq 3)$ obtained in the presence of one of the 27 individual candidates analyzed.

Additional file 2: Figure S1. Assessment of possible cytotoxic effects of candidate genes. 293T cells were transfected with $2.5 \mu \mathrm{g}$ of constructs expressing the candidate genes and the cell viability was examined two days later by MTT assay. Lack of cytotoxicity was confirmed in the CellTiter-Glo ${ }^{\circledast}$ Luminescent Cell Viability Assay. All data were normalized to the BFP only control construct (100\%) and are presented as mean \pm SD $(n=3)$. Figure S2. Assessment of antiviral effects of candidate genes. Impact of individual candidate factors on HIV-1 gene expression, release and infectivity. Candidates were ranked from the left to the right based on their inhibitory effect on the production of infectious wt HIV-1 (blue). Results obtained for the D3 virus (red) are shown for comparison. The detection limit for CA and CF p24 antigen in the ELISA assay is about 0.5\%. Figure S3. Correlation between the effect of the candidate genes on wt and D3 HIV-1 gene expression, virus release and viral infectivity. (A) Correlation between the effect of the candidate genes on wt and D3 HIV-1 in the indicated assays. Each symbol represents the average values $(n \geq 3)$ obtained in the presence of one of the 28 individual candidate genes analyzed. Candidate genes that reduced the values for both wt and D3 virus below 50\% (FACSbased assays) or below 10\% (p24 expression and infectious virus production) are color coded green; those that achieved this only for the D3 construct yellow; and enhancers of both viruses red. (B) Correlation between the effects of the candidate genes in the different assays for viral gene expression, p24 antigen production and viral infectivity. The upper panels give the results obtained for the wt HIV-1 construct and the lower panel provides the data generated using the D3 mutant construct.

Additional file 3: Table S2. Ranking of candidates in a relaxed screen. A set of 15,052 protein-coding genes was annotated with nine parameters informative for response to HIV-1 infection or evolutionary history. Parameters included in vivo response to HIV-1 infection, dNdS value, codon-specific positive selection, measurements of cross species adaptive evolution, burden of synonymous, missense and non-sense variation, intolerance to functional variation and number of paralogs. We highlight in yellow genes selected in the main screen, and in blue, pardigmatic restriction factors. 


\section{Competing interests}

The authors declare that they have no competing interests.

\section{Authors' contributions}

PJM, AG, NP, CK, DH, SFK, NG, JH, KM, provided the bulk of the experimental data presented. DT, JP, and MM contributed technical expertise and work. PJM, AR and AT performed bioinformatics analyses. DS and AC critically supervised the experimental work. PJM, FK and AT devised the experiments. FK and AT wrote the manuscript with support from DS, PJM and others. All authors read and approved the final manuscript.

\section{Acknowledgements}

We thank Gregory Jordan and Nadine Zangger for participation in early steps of the study. TZM-bl cells were obtained through the NIH AIDS Research and Reference Reagent Program. The study was supported by the Swiss National Science Foundation (AT) and by the Deutsche Forschungsgemeinschaft (Leibniz award to FK). DH and KM are funded by the International Graduate School in Molecular Medicine UIm. AT and FK were supported by European FP7 "HIT HIDDEN HIV" (305762) and an ERC Advanced grant to FK. The funders had no role in study design, data collection and analysis, decision to publish, or preparation of the manuscript. The authors declare no conflict of interest.

\section{Author details}

1'École Polytechnique Fédérale de Lausanne, 1015 Lausanne, Switzerland. ${ }^{2}$ Swiss Institute of Bioinformatics, 1005 Lausanne, Switzerland. ${ }^{3}$ Institute of Molecular Virology, Ulm University Medical Center, 89081 Ulm, Germany. ${ }^{4}$ Institute of Microbiology, University of Lausanne, 1011 Lausanne, Switzerland. ${ }^{5}$ J. Craig Venter Institute, La Jolla, CA 92037, USA.

\section{Received: 29 December 2014 Accepted: 24 February 2015} Published online: 16 May 2015

\section{References}

1. Daugherty MD, Malik HS. Rules of engagement: molecular insights from host-virus arms races. Annu Rev Genet. 2012;46:677-700.

2. Neil S, Bieniasz P. Human immunodeficiency virus, restriction factors, and interferon. J Interf Cytok Res. 2009;29:569-80

3. Rotger M, Dang KK, Fellay J, Heinzen EL, Feng S, Descombes $\mathrm{P}$, et al. Genome-wide mRNA expression correlates of viral control in CD4+ T-cells from HIV-1-infected individuals. PLoS Pathog. 2010;6, e1000781.

4. Malim MH, Emerman M. HIV-1 accessory proteins-ensuring viral survival in a hostile environment. Cell Host Microbe. 2008;3:388-98.

5. Kirchhoff F. Immune evasion and counteraction of restriction factors by HIV-1 and other primate lentiviruses. Cell Host Microbe. 2010;8:55-67.

6. Laguette $\mathrm{N}$, Benkirane M. How SAMHD1 changes our view of viral restriction. Trends Immunol. 2012;33:26-33.

7. Rotger M, Dalmau J, Rauch A, McLaren P, Bosinger SE, Marttinez R, et al. Comparative transcriptome analysis of extreme phenotypes of human HIV-1 infection and sooty mangabey and rhesus macaque models of SIV infection. J Clin Invest. 2011;121:2391-400.

8. Li Q, Smith AJ, Schacker TW, Carlis JV, Duan L, Reilly CS, et al. Microarray analysis of lymphatic tissue reveals stage-specific, gene expression signatures in HIV-1 infection. J Immunol. 2009;183:1975-82.

9. Berry MP, Graham CM, McNab FW, Xu Z, Bloch SA, Oni T, et al. An interferon-inducible neutrophil-driven blood transcriptional signature in human tuberculosis. Nature. 2010:466:973-7.

10. Thompson LJ, Dunstan SJ, Dolecek C, Perkins T, House D, Dougan G, et al. Transcriptional response in the peripheral blood of patients infected with Salmonella enterica serovar Typhi. Proc Natl Acad Sci U S A. 2009;106:22433-8.

11. Long HT, Hibberd ML, Hien TT, Dung NM, Van NT, Farrar J, et al. Patterns of gene transcript abundance in the blood of children with severe or uncomplicated dengue highlight differences in disease evolution and host response to dengue virus infection. J Inf Dis. 2009;199:537-46.

12. Ubol S, Masrinoul P, Chaijaruwanich J, Kalayanarooj S, Charoensirisuthikul T, Kasisith J. Differences in global gene expression in peripheral blood mononuclear cells indicate a significant role of the innate responses in progression of dengue fever but not dengue hemorrhagic fever. J Inf Dis. 2008;197:1459-67.

13. Yang Z. Likelihood ratio tests for detecting positive selection and application to primate lysozyme evolution. Mol Biol Evol. 1998;15:568-73.
14. Kosiol C, Vinar T, da Fonseca RR, Hubisz MJ, Bustamante CD, Nielsen R, et al. Patterns of positive selection in six Mammalian genomes. PLoS Genet. 2008;4, e1000144.

15. Jager S, Cimermancic P, Gulbahce N, Johnson JR, McGovern KE, Clarke SC, et al. Global landscape of HIV-human protein complexes. Nature. 2012;481:365-70.

16. Yang Z. PAML: a program package for phylogenetic analysis by maximum likelihood. Comput Appl Biosci. 1997;13:555-6.

17. Ortiz M, Guex N, Patin E, Martin O, Xenarios I, Ciuffi A, et al. Evolutionary trajectories of primate genes involved in hiv pathogenesis. Mol Biol Evol. 2009;26:2865-75.

18. Laguette N, Rahm N, Sobhian B, Chable-Bessia C, Münch J, Snoeck J, et al. Evolutionary and functional analyses of the interaction between SAMHD1 and the lentiviral Vpx protein. Cell Host Microbe. 2012;11:205-17.

19. Schindler M, Wurfl S, Benaroch P, Greenough TC, Daniels R, Easterbrook P, et al. Down-modulation of mature major histocompatibility complex class II and up-regulation of invariant chain cell surface expression are well-conserved functions of human and simian immunodeficiency virus nef alleles. J Virol. 2003;77:10548-56.

20. Rausell A, McLaren PJ, Telenti A. p. F1000 Prime Rep. 2013;5:29.

21. Harris RS, Hultquist JF, Evans DT. The restriction factors of human immunodeficiency virus. J Biol Chem. 2012;287:40875-83.

22. Meyerson NR, Rowley PA, Swan CH, Le DT, Wilkerson GK, Sawyer SL. Positive selection of primate genes that promote HIV-1 replication. Virology. 2014;454-455:291-8.

23. Smith EE, Malik HS. The apolipoprotein L family of programmed cell death and immunity genes rapidly evolved in primates at discrete sites of host-pathogen interactions. Genome Res. 2009;19:850-8.

24. Vanhamme L, Paturiaux-Hanocq F, Poelvoorde P, Nolan DP, Lins L, Van Den Abbeele J, et al. Apolipoprotein L-I is the trypanosome lytic factor of human serum. Nature. 2003:422:83-7.

25. Schoggins JW, Wilson SJ, Panis M, Murphy MY, Jones CT, Bieniasz P, et al. A diverse range of gene products are effectors of the type I interferon antiviral response. Nature. 2011;472:481-5.

26. Forde S, Tye BJ, Newey SE, Roubelakis M, Smythe J, McGuckin CP, et al. Endolyn (CD164) modulates the CXCL12-mediated migration of umbilical cord blood CD133+ cells. Blood. 2007;109:1825-33.

27. Hovanessian AG. On the discovery of interferon-inducible, double-stranded RNA activated enzymes: the 2'-5'oligoadenylate synthetases and the protein kinase PKR. Cytokine Growth Factor Rev. 2007;18:351-61.

28. Jakobsen MR, Bak RO, Andersen A, Berg RK, Jensen SB, Tengchuan J, et al. IFI16 senses DNA forms of the lentiviral replication cycle and controls HIV-1 replication. Proc Natl Acad Sci U S A. 2013;110:E4571-80.

29. Orzalli MH, Knipe DM. Cellular sensing of viral DNA and viral evasion mechanisms. Annu Rev Microbiol. 2014;68:477-92.

30. Monroe KM, Yang Z, Johnson JR, Geng X, Doitsh G, Krogan NJ, et al. IFI16 DNA sensor is required for death of lymphoid CD4 T cells abortively infected with HIV. Science. 2014;343:428-32.

31. Doitsh G, Galloway NL, Geng X, Yang Z, Monroe KM, Zepeda O, et al. Cell death by pyroptosis drives CD4 T-cell depletion in HIV-1 infection. Nature. 2014;505:509-14

32. Pertel T, Hausmann S, Morger D, Zuger S, Guerra J, Lascano J, et al. TRIM5 is an innate immune sensor for the retrovirus capsid lattice. Nature. 2011;472:361-5.

33. Galao RP, Le Tortorec A, Pickering S, Kueck T, Neil SJ. Innate sensing of HIV-1 assembly by Tetherin induces NFkappaB-dependent proinflammatory responses. Cell Host Microbe. 2012;12:633-44.

34. Hotter D, Sauter D, Kirchhoff F. Emerging role of the host restriction factor tetherin in viral immune sensing. J Mol Biol. 2013;425:4956-64.

35. Galao RP, Pickering S, Curnock R, Neil SJ. Retroviral retention activates a Syk-dependent HemITAM in human tetherin. Cell Host Microbe. 2014;16:291-303.

36. Roscioli T, Cliffe ST, Bloch DB, Bell CG, Mullan G, Taylor PJ, et al. Mutations in the gene encoding the PML nuclear body protein Sp110 are associated with immunodeficiency and hepatic veno-occlusive disease. Nat Genet. 2006;38:620-2

37. Nicewonger J, Suck G, Bloch D, Swaminathan S. Epstein-Barr virus (EBV) SM protein induces and recruits cellular Sp110b to stabilize mRNAs and enhance EBV lytic gene expression. J Virol. 2004;78:9412-22.

38. Lajoie J, Juno J, Burgener A, Rahman S, Mogk K, Wachihi C, et al. A distinct cytokine and chemokine profile at the genital mucosa is associated with 
HIV-1 protection among HIV-exposed seronegative commercial sex workers. Mucosal Immunol. 2012;5:277-87.

39. Kim BH, Shenoy AR, Kumar P, Bradfield CJ, MacMicking JD. IFN-inducible GTPases in host cell defense. Cell Host Microbe. 2012;12:432-44.

40. Vestal DJ, Jeyaratnam JA. The guanylate-binding proteins: emerging insights into the biochemical properties and functions of this family of large interferon-induced guanosine triphosphatase. J Interferon Cytokine Res. 2011;31:89-97.

41. Lu J, Pan Q, Rong L, He W, Liu SL, Liang C. The IFITM proteins inhibit HIV-1 infection. J Virol. 2011;85:2126-37.

42. Qian J, Le Duff Y, Wang Y, Pan Q, Ding S, Zheng YM, et al. Primate lentiviruses are differentially inhibited by interferon-induced transmembrane proteins. Virology. 2015;474:10-8.

43. Lefebvre JC, Giordanengo V, Limouse M, Doglio A, Cucchiarini M, Monpoux F, et al. Altered glycosylation of leukosialin, CD43, in HIV-1-infected cells of the CEM line. J Exp Med. 1994;180:1609-17.

44. Gallego MD, Aguado E, Kindelan JM, Pena J, Santamaria M, Molina IJ. Altered expression of CD43-hexasaccharide isoform on peripheral $\mathrm{T}$ lymphocytes from HIV-infected individuals. AIDS. 2001;15:477-81.

45. Sandler NG, Bosinger SE, Estes JD, Zhu RT, Tharp GK, Boritz E, et al. Type interferon responses in rhesus macaques prevent SIV infection and slow disease progression. Nature. 2014;511:601-5.

46. Telenti A. The mixed blessing of interferon. Nature. 2014;511:537-8.

47. Fellay J, Shianna KV, Ge D, Colombo S, Ledergerber B, Weale M, et al. A whole-genome association study of major determinants for host control of HIV-1. Science. 2007:317:944-7.

48. Vilella AJ, Severin J, Ureta-Vidal A, Heng L, Durbin R, Birney E. EnsemblCompara GeneTrees: Complete, duplication-aware phylogenetic trees in vertebrates. Genome Res. 2009;19:327-35.

49. Schneider A, Souvorov A, Sabath N, Landan G, Gonnet GH, Graur D. Estimates of positive Darwinian selection are inflated by errors in sequencing, annotation, and alignment. Gen Biol Evol. 2009;1:114-8.

50. Paten B, Herrero J, Beal K, Fitzgerald S, Birney E. Enredo and Pecan: genome-wide mammalian consistency-based multiple alignment with paralogs. Genome Res. 2008;18:1814-28.

51. Wong WS, Yang Z, Goldman N, Nielsen R. Accuracy and power of statistical methods for detecting adaptive evolution in protein coding sequences and for identifying positively selected sites. Genetics. 2004;168:1041-51.

52. Benjamini $Y$, Hochberg Y. Controlling the false discovery rate : a practical and powerful approach to multiple testing. J Royal Strat Soc. 1995;57:289-300.

53. Sauter D, Schindler M, Specht A, Landford WN, Munch J, Kim KA, et al. Tetherin-driven adaptation of Vpu and Nef function and the evolution of pandemic and nonpandemic HIV-1 strains. Cell Host Microbe. 2009;6:409-21.

54. Schindler M, Munch J, Kutsch O, Li H, Santiago ML, Bibollet-Ruche F, et al. Nef-mediated suppression of t cell activation was lost in a lentiviral lineage that gave rise to HIV-1. Cell. 2006;125:1055-67.

55. Wildum S, Schindler M, Munch J, Kirchhoff F. Contribution of Vpu, Env, and Nef to CD4 down-modulation and resistance of human immunodeficiency virus type 1-infected T cells to superinfection. J Virol. 2006;80:8047-59.

56. Martin M. Cutadapt removes adapter sequences from high-throughput sequencing reads. EMBnetjournal. 2011;17

57. Schmieder R, Edwards R. Quality control and preprocessing of metagenomic datasets. Bioinformatics. 2011;27:863-4.

58. Charlesworth J, Eyre-Walker A. The McDonald-Kreitman test and slightly deleterious mutations. Mol Biol Evol. 2008;25:1007-15.

59. Petrovski S, Wang Q, Heinzen EL, Allen AS, Goldstein DB. Genic intolerance to functional variation and the interpretation of personal genomes. PLoS Genet. 2013;9, e1003709. 